\title{
Mesenteritis esclerosante: diagnóstico y tratamiento
}

\author{
Sclerosing mesenteritis: Diagnosis and treatment

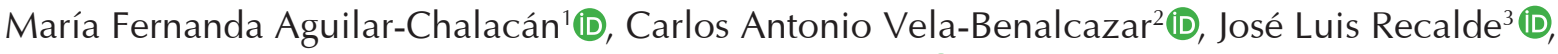 \\ Ricardo Negrete $^{2} \mathbb{D}$ \\ 1 Médico, especialista en Cirugía general y laparoscópica, Pontificia Universidad Católica del Ecuador, Quito, Ecuador. \\ 2 Médico, especialista en Cirugía general y laparoscópica, Hospital Vozandes Quito, Ecuador. \\ 3 Médico, especialista en Cirugía general y laparoscópica, Hospital Vozandes, Quito, Ecuador.
}

\section{Resumen}

Introducción. El objetivo de este artículo es dar a conocer el caso de un paciente con diagnóstico de mesenteritis esclerosante quien cursó con cuadro de obstrucción intestinal.

Descripción del caso. Paciente masculino de 28 años de edad, quien se presenta con cuadro clínico sugestivo de obstrucción intestinal, por lo que se decide resolución quirúrgica. Durante la cirugía se observa una zona fibrótica y adherente del intestino delgado, asociada a un mesenterio engrosado. El exámen histopatológico de la pieza quirúrgica confirmó el diagnóstico de mesenteritis esclerosante.

Discusión. La mesenteritis esclerosante es una patología de baja incidencia, y su forma de presentación es inespecífica, por lo que el diagnóstico definitivo es histopatológico. La tomografía es útil para el diagnóstico cuando se tiene la sospecha clínica. Puede optarse por el tratamiento quirúrgico para los casos que se presenten con clínica de obstrucción intestinal, o en caso contrario, el tratamiento médico a base de fármacos inmunosupresores e inmunomoduladores ha demostrado ser efectivo. De acuerdo con los diferentes estudios publicados hasta el momento, se observa una adecuada respuesta, independientemente del tratamiento empleado.

Palabras clave: obstrucción; intestinal; mesenteritis; esclerosante; retráctil; lipodistrofia.

\begin{abstract}
Introduction. The objective of this article is to present the case of a patient diagnosed with sclerosing mesenteritis who presented with intestinal obstruction.

Case description. A 28-year-old male patient, who presented with a clinical picture suggestive of intestinal obstruction, for which a surgical resolution was decided. During surgery, a fibrotic and adherent area of the small intestine is observed, associated with a thickened mesentery. The histopathological examination of the surgical specimen confirmed the diagnosis of sclerosing mesenteritis.
\end{abstract}

Fecha de recibido: 03/07/2020 - Fecha de aceptación: 21/08/2020 - Fecha de publicación en línea: 05/03/2021

Correspondencia: María Fernanda Aguilar-Chalacán. Código Postal: 171101. Quito, Ecuador. Teléfono: (+593) 0989015148

Correo electrónico: feraguilar88@hotmail.com

Citar como: Aguilar-Chalacán MF, Vela-Benalcazar CA, Recalde JL, Negrete R. Mesenteritis esclerosante: diagnóstico y tratamiento. Rev Colomb Cir. 2021;36:338-43. https://doi.org/10.30944/20117582.707

Este es un artículo de acceso abierto bajo una Licencia Creative Commons - BY-NC-ND https://creativecommons.org/licenses/by-ncnd/4.0/deed.es 
Discussion. Sclerosing mesenteritis is a low incidence pathology, and its presentation is nonspecific, so the definitive diagnosis is histopathological. Tomography is useful for diagnosis when there is clinical suspicion. Surgical treatment can be chosen for cases that present with symptoms of intestinal obstruction, or otherwise medical treatment based on immunosuppressive and immunomodulatory drugs has proven to be effective. According to the different studies published so far, an adequate response is observed, regardless of the treatment used.

Keywords: obstruction; intestinal; mesenteritis; sclerosing; retractable; lipodystrophy.

\section{Introducción}

El término mesenteritis esclerosante, hace referencia a una enfermedad idiopática, rara, de curso crónico, caracterizada por cambios inflamatorios y fibróticos en el tejido adiposo del mesenterio del intestino ${ }^{1}$. Según Sharma en su revisión sistemática, fue descrita por primera vez por Jura en 1924 como mesenteritis retráctil; posteriormente Crane, en 1955, presenta cinco casos con mesenterio intestinal firme y engrosado, y Ogden en 1960 la reseña como una paniculitis mesentérica. Con el advenimiento de la tomografía, Mindelzun describe los hallazgos tomográficos conocidos como "mesenterio brumoso" y en 1980, Cherayil analiza la composición lipídica del mesenterio ${ }^{1}$.

No se conocen estudios que determinen la prevalencia de la enfermedad en la población general, pero en algunos se estima la frecuencia de la mesenteritis esclerosante en grandes series de pacientes consecutivos, a los que se les realizó tomografía por otros motivos. La frecuencia en estas series oscila entre el $0,1 \%$ y el $7,8 \%^{2}$. Se ha relacionado con una gran variedad de causas como cirugía abdominal previa, trauma, causas autoinmunitarias o patología oncológica, que puede estar presente al momento del diagnóstico o desarrollarse después del mismo; el linfoma, es la neoplasia más frecuentemente asociada ${ }^{3}$. La mesenteritis, ocurre más en varones, y la edad promedio de presentación es en la sexta década de la vida ${ }^{1,3}$.

Clínicamente se presenta con signos y síntomas inespecíficos, siendo el dolor abdominal la principal manifestación clínica, además de distensión abdominal y la presencia de una masa abdominal, que muchas veces puede confundirse con un tumor, lo que dificulta el diagnóstico ${ }^{1}$. El patrón de oro para el diagnóstico es la biopsia con el análisis histopatológico ${ }^{1}$.
El tratamiento puede ser médico, con el uso de fármacos inmunosupresores e inmunomoduladores, o quirúrgico, siendo la obstrucción intestinal la indicación más frecuente para cirugía ${ }^{4}$.

\section{Métodos}

Realizamos una revisión bibliográfica de mesenteritis esclerosante en Medline, Pubmed, Google académico y Tripdatabase, siendo pocos los casos reportados hasta la fecha, y limitándose la mayoría de las publicaciones a series de casos.

\section{Caso clínico}

Paciente masculino de 28 años de edad, con antecedente de laparotomía exploratoria y resección intestinal por obstrucción intestinal al mes de vida. El paciente ingresó con cuadro de 12 horas de evolución de dolor abdominal, localizado en epigastrio, asociado a vómito. Al examen físico, los signos vitales eran normales, el abdomen distendido, doloroso a la palpación profunda en epigastrio e hipocondrio izquierdo. En el hemograma se encontró leucocitosis de 12.070 células por microlitro y neutrofilia de $84 \%$. Se realizó una tomografía computarizada de abdomen que informó distensión de asas de intestino delgado, con zona de transición a nivel del íleon terminal, compatible con obstrucción intestinal mecánica (posible brida intestinal) (Figura1).

Por lo anterior, el paciente fue diagnosticado con obstrucción intestinal por adherencias y se realizó una liberación de adherencias por laparoscopia. En el posoperatorio, el paciente se mantuvo con débitos altos por la sonda nasogástrica, por lo que al quinto día se solicitó un tránsito intestinal con gastrografina, sin existir progresión del contraste en el intestino delgado, con falta de vaciamiento gástrico (Figura 2). 
Posteriormente se decidió una nueva intervención quirúrgica y se realizó laparotomía exploratoria, identificando un asa de intestino delgado con zona de estenosis aproximadamente a 150 cm de la válvula ileocecal (Figura 3), que se trató mediante resección intestinal mas anastomosis. El examen histopatológico reveló mesenteritis esclerosante. A nivel de la subserosa y meso del intestino delgado, se observó una marcada proliferación en sábanas de fibroblastos fusiformes y estrellados (Figura 4). También se observaron macrófagos cargados de lípidos junto a áreas hipercelulares con mitosis frecuentes y zonas de colágeno gruesos en medio de los fibroblastos, edema estromal e inflamación linfoplasmocitaria (Figura 5).

\section{Discusión}

La mesenteritis esclerosante es una condición rara, caracterizada por la inflamación del mesenterio, que puede ser, desde una inflamación aguda, hasta un proceso fibrótico crónico. Es más frecuente en hombres entre 50 y 60 años, y en un $50 \%$ de los casos, el diagnóstico es incidental y se realiza mediante el estu dio histológico de la biopsia ${ }^{5}$.

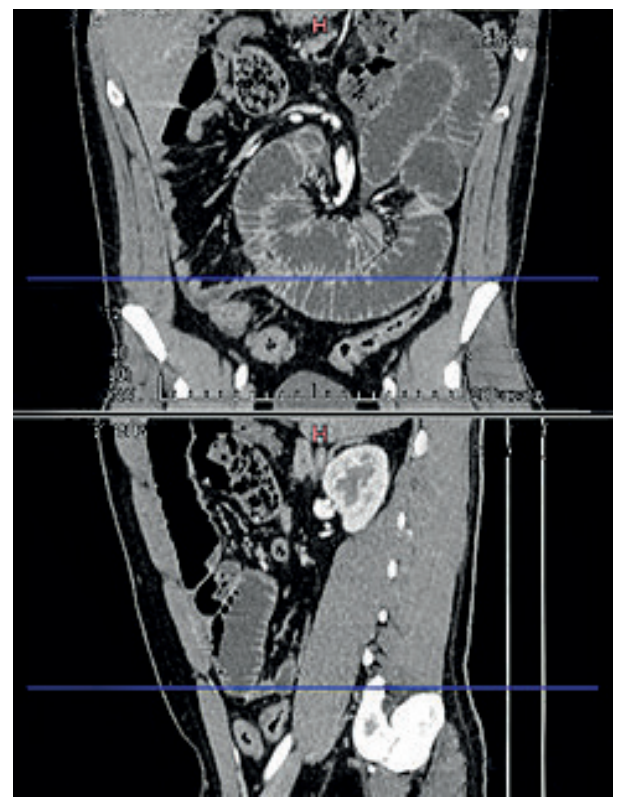

Figura 1. Zona de transición a nivel de íleon

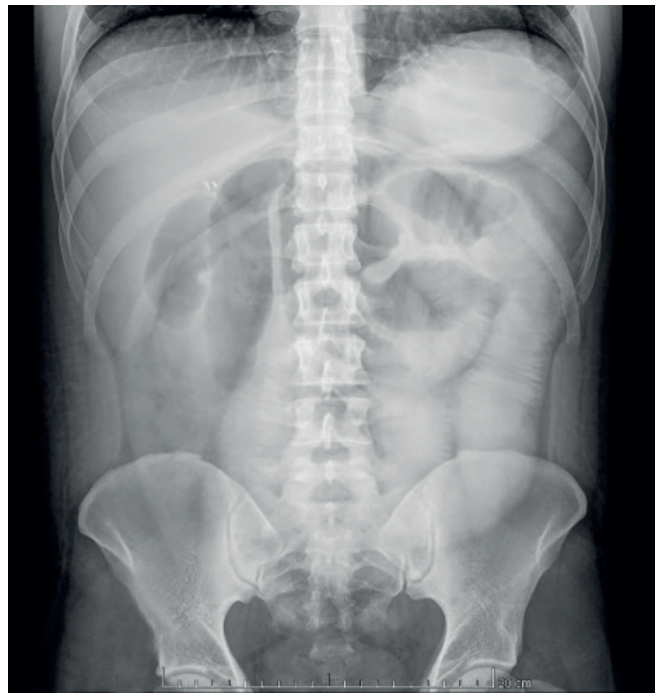

Figura 2. Imagen radiológica que muestra la falta de progresión del medio de contraste.

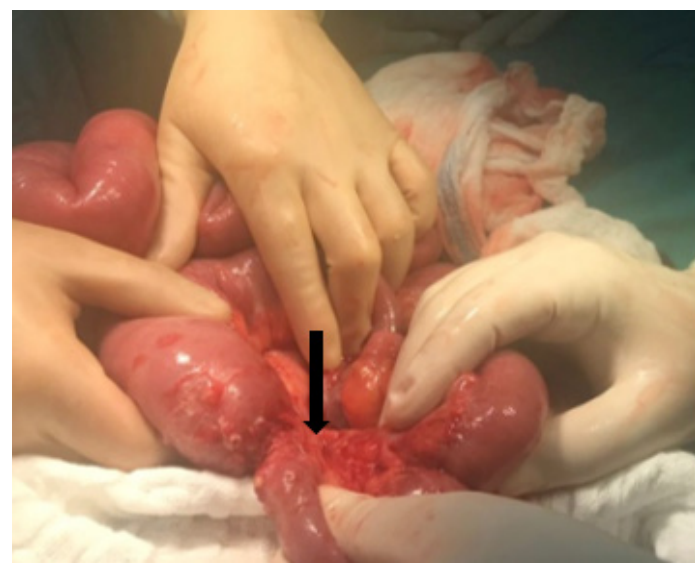

Figura 3. Zona de estenosis en el intestino delgado.

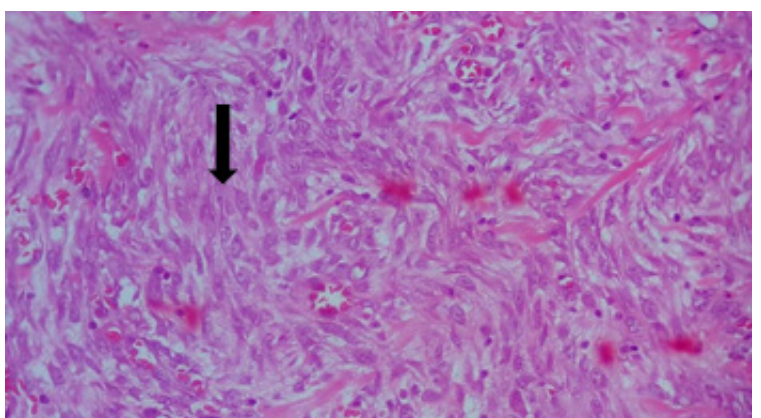

Figura 4. Marcada proliferación de fibroblastos en la subserosa y meso del intestino delgado (hematoxilinaeosina 10X). 


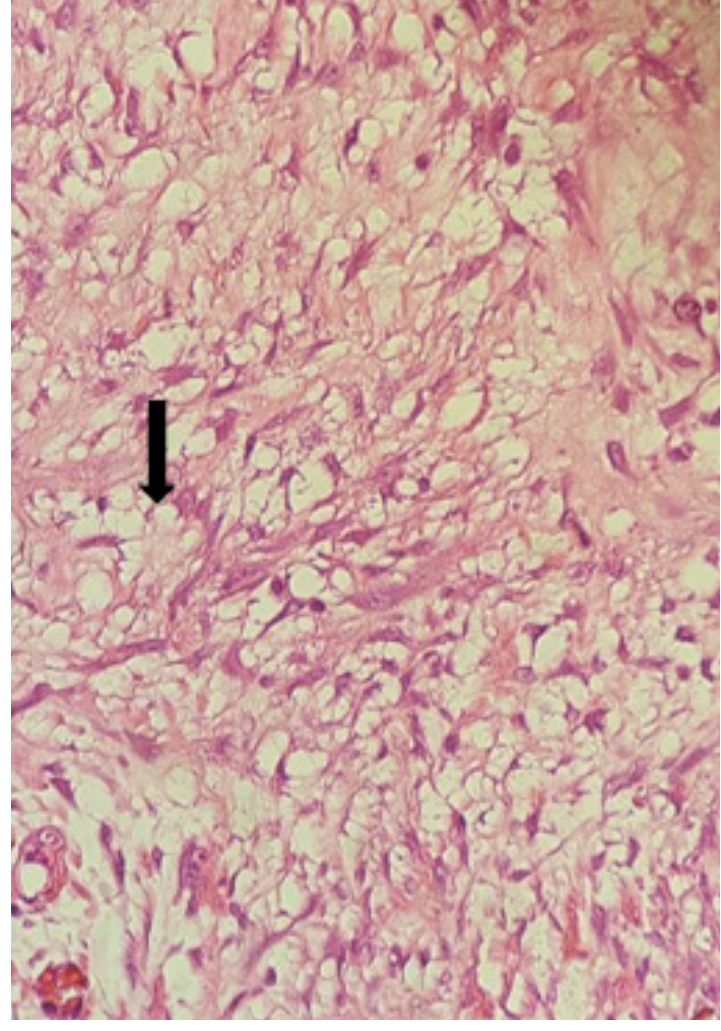

Figura 5. Lipodistrofia del mesenterio: macrófagos espumosos cargados de lípidos junto a fibras de colágeno (hematoxilina-eosina 20X).

Es un trastorno fibro-inflamatorio, de etiología desconocida, que afecta principalmente al mesenterio del intestino delgado ${ }^{5-7}$. Se describen diferentes tipos histológicos en relación a la evolución de la enfermedad: lipodistrofia mesentérica con predominio de macrófagos espumosos cargados de lípidos, paniculitis mesentérica en donde existe un predominio de células plasmáticas y linfocitos, y la mesenteritis retráctil, la fase final, en donde predomina la fibrosis por el depósito de colágeno ${ }^{7}$.

Hasta la fecha no se ha podido determinar una causa específica, se ha implicado una amplia variedad de etiologías asociadas al desarrollo de mesenteritis esclerosante. Hasta en el 58,8 \% de los casos no existe una causa desencadenante ${ }^{2,7}$.

En una revisión sistemática publicada por Halligan S, et al., se encontró que hasta el $43 \%$ de los pacientes tenían algún antecedente quirúrgico, siendo la apendicectomía la cirugía más frecuente, y el $38 \%$ tenían enfermedad maligna al momento del diagnóstico, siendo el linfoma la neoplasia más frecuente, hasta en el $25 \%$ de los casos. El $6,4 \%$ de los pacientes desarrollaron enfermedad maligna posterior al diagnóstico, principalmente linfoma en el $33 \%$ de los casos ${ }^{3}$.

Es más frecuente en hombres $(69,3 \%)$ y con un pico de presentación en la sexta década de la vida, siendo la etnia caucásica la más prevalente $(60,8 \%)$. Dichos resultados son similares a los encontrados por Gutiérrez, quien además describe entre los antecedentes personales, hipertensión arterial en el $10 \%$ de los pacientes ${ }^{1,2,6}$.

La presentación clínica es inespecífica, con signos y síntomas que pueden sugerir otras patologías, y con duración de más de un mes ${ }^{1}$. Los autores describen con mayor frecuencia dolor abdominal (78,1\%), sensibilidad (38\%) y la presencia de una masa a la palpación abdominal $(39,5 \%)^{1,2,6}$.

Con relación a los hallazgos de laboratorio, no existen exámenes específicos de esta patología, pero se puede encontrar un aumento de las proteínas de fase aguda, proteína $C$ reactiva (PCR) en el 86,5 \% y velocidad de sedimentación globular (VSG) en el 88,4 \%; sin embargo, si no se tiene la sospecha clínica, estos estudios no ayudarán al diagnóstico ${ }^{1}$.

El diagnóstico por imágenes todavía sigue siendo un reto, ya que a pesar de que hay hallazgos imagenológicos típicos, muchas veces es difícil identificarlos, salvo que se tenga la sospecha clínica ${ }^{8}$. La tomografía es la que tiene mayor sensibilidad y especificidad; existe una serie de signos característicos, que en conjunto se conocen como "mesenterio brumoso". Hasta en el $85 \%$ de los casos se observa el signo del "halo graso", que se caracteriza por la presencia de un anillo de grasa hipodensa alrededor de los vasos, pero también se pueden encontrar nódulos o una masa mesentérica ${ }^{9}$.

En el $59 \%$ de los casos se puede encontrar una "pseudocápsula tumoral", que corresponde a una banda densa de tejido blando (menor de $3 \mathrm{~mm}$ ), separando la grasa normal alrededor del mesenterio inflamado. En el $80 \%$ se pueden encontrar nódulos de tejido blando (menores de $5 \mathrm{~mm}$ ) dentro 
del mesenterio inflamado; en este caso el tamaño del nódulo es importante ya que para los nódulos mayores de $10 \mathrm{~mm}$ deben tomarse biopsias, para establecer el diagnóstico diferencial con patología neoplásica ${ }^{10}$.

Otra opción diagnóstica y mínimamente invasiva que puede emplearse en pacientes con sospecha de mesenteritis esclerosante, es la biopsia con aguja guiada por tomografía o ultrasonografía, que permite obtener tejido mesentérico para el estudio histopatológico ${ }^{11}$.

Existen varias opciones terapéuticas, sin embargo, la mayoría son empíricas y provienen de casos individuales, sin el uso de medidas que determinen la severidad de los síntomas ${ }^{4}$. Akram $S$, et al., encontraron que el tratamiento médico se empleó en el $56 \%$ de los casos, y de este grupo, en el $63 \%$ se administró tamoxifeno más prednisolona, con $60 \%$ de respuesta al tratamiento luego de un periodo de 12 a 16 semanas, pero $30 \%$ presentaron síntomas persistentes y en el $10 \%$ hubo progresión de la enfermedad sin mejoría clínica. En el $45 \%$ de los casos se empleó el tratamiento quirúrgico, siendo la indicación más frecuente la obstrucción intestinal (70 \% de los casos), como en el caso que se presenta. Los procedimientos practicados fueron resección segmentaria del intestino debido al compromiso vascular (57\%), baipás paliativo (21\%) y solo liberación de adherencias $(21 \%)^{6}$. Es importante notar que, independientemente del tratamiento empleado, en un mes de seguimiento se observó respuesta al tratamiento con resolución de los síntomas y de los hallazgos imagenológicos en el $86 \%$ de los casos ${ }^{6}$.

Resultados similares reveló el estudio de Sharma P, et al., en donde el 34,9\% fueron sometidos a tratamiento médico, y dentro de este grupo en el 56,8 \% de los casos se usó corticoides. El tratamiento quirúrgico se empleó en el 41,7 \% y el tratamiento conservador a base de hidratación y analgesia en el 17,2 \%. Durante el seguimiento que duro 10 meses, se observó resolución de los síntomas y de los hallazgos imagenológicos en el $78,6 \%$ y no hubo cambios en el $10,9 \%{ }^{1}$. Gutiérrez reportó una tasa de respuesta completa al tratamiento del 80,8 \% de los pacientes ${ }^{2}$.

\section{Conclusiones}

La mesenteritis esclerosante, es una patología de baja incidencia y su forma de presentación es inespecífica, manifestándose muchas veces como un cuadro clínico de obstrucción intestinal que requiere intervención quirúrgica, por lo que el diagnóstico definitivo es histopatológico. La tomografía es útil para el diagnóstico cuando se tiene la sospecha clínica y se buscan signos característicos de mesenteritis esclerosante.

Puede optarse por el tratamiento quirúrgico para los casos que se presenten con clínica de obstrucción intestinal, como nuestro paciente. En caso contrario, el tratamiento médico, a base de fármacos inmunosupresores e inmunomoduladores, ha demostrado ser efectivo. En los diferentes estudios publicados hasta el momento se encuentra una adecuada respuesta, independientemente del tratamiento empleado.

\section{Cumplimiento de normas éticas}

Consentimiento informado: Este artículo es la descripción retrospectiva de un caso clínico y no implicó riesgo alguno para el paciente, por lo que no se requiere consentimiento informado.

Conflicto de interés: Ninguno declarado por los autores.

Fuente de financiación: Financiado con recursos propios de los autores.

\section{Contribución de los autores:}

Concepción y diseño del estudio: Fernanda Aguilar, José Luis Recalde, Ricardo Negrete.

Adquisición de datos: Fernanda Aguilar, José Luis Recalde, Ricardo Negrete.

Análisis e interpretación de datos: Carlos Vela, Fernanda Aguilar.

Redacción del manuscrito: Fernanda Aguilar.

Revisión crítica: Carlos Vela, José Luis Recalde, Ricardo Negrete.

\section{Referencias}

1. Sharma P, Yadav S, Needham CM, Feuerstadt P. Sclerosing mesenteritis: A systematic review of 192 cases. Clin J Gastroenterol. 2017;10:103-11. https://doi.org/10.1007/s12328-017-0716-5. 
2. Del Mar Ayala Gutiérrez M, De Ramón Garrido E. Paniculitis mesentérica. Med Clin (Barc). 2016;146:497505. https://doi.org/10.1016/j.medcli.2016.01.015.

3. Halligan S, Plumb A, Taylor S. Mesenteric panniculitis: A systematic review of cross-sectional imaging findings and risk of subsequent malignancy. Eur Radiol. 2016;26:4531-7. https://doi.org/10.1007/s00330-016-4298-2.

4. Hussein MRA, Abdelwahed SR. Mesenteric panniculitis: An update. Expert Rev Gastroenterol Hepatol. 2015;9:67-78. https://doi.org/10.1586/17474124.2014.939632.

5. González P, Castro J, Rojas G, Ramírez C, Toiber M. Mesenteritis esclerosante. Presentación de tres casos y revisión de la literatura. Cir Ciruj. 2008;76:343-8.

6. Akram S, Pardi DS, Schaffner JA, Smyrk TC. Sclerosing mesenteritis: clinical features, treatment, and outcome in ninety-two patients. Clin Gastroenterol Hepatol. 2007;5:589-96.

https://doi.org/10.1016/j.cgh.2007.02.032.
7. Hiroshi K. An autopsy case of mesenteric panniculitis with massive pleural effusions. Am J Case Reports. 2018;19:13-20.

https://doi.org/10.12659/AJCR.905744.

8. Beyer LP, Schreyer A. "Misty mesentery". Radiologe. 2018;58:56-9.

https://doi.org/10.1007/s00117-017-0330-z.

9. Zhang P, Dyer RB. The "fat ring" sign of sclerosing mesenteritis. Abdom Radiol. 2018;43:1839-40. https://doi.org/10.1007/s00261-017-1353-7.

10. Van Breda Vriesman AC, Schuttevaer HM, Coerkamp EG, Puylaert JBCM. Mesenteric panniculitis: US and CT features. Eur Radiol. 2004;14:2242-8. https://doi.org/10.1007/s00330-004-2429-7.

11. Ueno M, Nishimura N, Shimodate Y, Doi A, Mouri H, Matsueda K, et al. Sclerosing mesenteritis diagnosed with computed tomography and ultrasound-guided needle biopsy: the utility of the coaxial technique. Clin J Gastroenterol. 2018;11:92-5. https://doi.org/10.1007/s12328-017-0800-x. 